Published in final edited form as:

Curr Opin Lipidol. 2009 June ; 20(3): 190-196. doi:10.1097/MOL.0b013e32832d3a10.

\title{
ApoE Receptors in the Nervous System
}

\author{
Joachim Herz \\ Department of Molecular Genetics, UT Southwestern, Dallas, TX 75390-9046
}

\begin{abstract}
Purpose of review-Over the last few years ApoE receptors, also known as LDL receptor related proteins, have distinguished themselves as functionally diverse signaling receptors with pivotal roles not only in the vascular system, but also in the nervous system and during development.

Recent findings-The expanding roles of ApoE receptors for cellular signal transduction at the same time transcend and integrate their lipid transport roles into a larger biological and clinical context. ApoE receptors are essential for the development of the nervous system, the regulation of synaptic plasticity, neuroprotection, and the innervation of the muscle. They also regulate the metabolism of the amyloid precursor protein on multiple levels, implicating them in the pathogenesis of Alzheimer's disease (AD).
\end{abstract}

Summary-ApoE, a common ligand for all members of the evolutionarily ancient LDL receptor gene family, is the major genetic modifier of the age of onset of Alzheimer's disease. The underlying molecular mechansims remain shrouded in mystery, but the numerous critical functions of ApoE receptors within and outside the nervous system that have recently emerged make it likely that these multifunctional signal modulators participate in AD pathogenesis. This review attempts to summarize the most recent and relevant findings in this area.

\section{Keywords}

LDL receptor family; dementia; synaptic plasticity; neuronal survival

\section{Introduction}

The last decade has seen a role reversal for lipoprotein receptors, and in particular the LDL receptor related proteins (LRPs, Figure 1). Rather than being perceived as mere transporters of lipoproteins, lipids and other macromolecules in the circulation, these multifunctional and evolutionarily ancienct cell surface proteins were first recognized for what initially appeared to be isolated and specialized functions as intercellular signal transducers. More recently, this image has further evolved to reveal that ApoE receptors cannot only act as direct signal transducers, but that they function also on a much broader scale as signal modulators as well as integrators of several distinct and independent signaling pathways. Here I am going to review the most recent findings with particular emphasis on the role of ApoE receptors in the nervous system.

\footnotetext{
Publisher's Disclaimer: This is a PDF file of an unedited manuscript that has been accepted for publication. As a service to our customers we are providing this early version of the manuscript. The manuscript will undergo copyediting, typesetting, and review of the resulting proof before it is published in its final citable form. Please note that during the production process errors may be discovered which could affect the content, and all legal disclaimers that apply to the journal pertain.
} 


\section{Control of Brain Development and Architecture}

That ApoE receptors have critical functions during brain development became apparent during the analysis of knockout mice lacking megalin/LRP2, and from animals deficient for both Apoer2/LRP8 and Vldlr. The latter are phenotypically identical to mice lacking the developmental signaling protein Reelin and to another strain of mice carrying mutations in the cytoplasmic adapter protein Disabled-1 (Dab1). This immediately suggested that both of these ApoE receptors function together in a linear signaling pathway that is dependent upon the extracellular ligand Reelin and the intracellular adaptor Dab1 for initiating a signaling cascade that regulates the migration and positioning of neurons during development [1]. The power of mouse genetics has been invaluable for the initial discovery of the basic frame work of this essential and evolutionarily highly conserved signaling pathway (Figure 2). This subsequently allowed for the rapid elucidation of the intracellular branches of a signaling cascade that is initiated by Reelin induced receptor clustering and followed by the activation of Src family tyrosine kinases (SFKs) through proximity triggered transphosphorylation. These include activation of PI3 kinase, Akt and inhibition of GSK3 $\beta$ resulting in reduced phosphorylation of the microtubule stabilizing protein tau, regulation of microtubule dynamics through Reelin triggered interaction of Dab1 with Lis1 (Pafah1b1), direct interaction of the Pafah1b complex with Vldlr [2], interaction of phosphorylated Dab1 with the adapter proteins Crk and CrkL [3,4], and stabilization of the actin cytoskeleton of neuronal processes by phosphorylation of the actin depolymerizing protein N-cofilin [5]. Recently, Thrombospondin-1 has been recognized as a novel ligand for Apoer 2 and Vldlr in the brain where it participates in the chain migration of postnatal neurons along the rostral migratory stream [6].

These novel interactions, which take place downstream of, or in parallel with, the activation of SFKs and which are partly dependent upon unique interaction motifs within the cytoplasmic domains of the receptors help to explain the specific and non-redundant roles Apoer2 and Vldlr have in shaping different regions of the brain in response to the binding of the same ligand or ligands. For instance, Apoer2 and Vldlr play different roles in the patterning of the cerebellum, in particular the topography of the Purkinje cell layer [7]. Similarly, in the neocortex Vldlr provides a stop signal for migrating neurons, preventing them from entering the marginal zone, while Apoer2 regulates the migration of later-born neurons and is essential for proper lamination of neocortex and hippocampus [8]. Intriguingly, regulation of neuronal migration by Reelin also involves interaction with Notch proteins, where stabilization of the intracellular (signaling) domain of Notch requires Reelin and Dab1 [9]. Reelin signaling through Apoer2 and Vldlr is also necessary for neuronal positioning in the spinal cord and for the processing of nociceptive input, although the alternative splicing of the Apoer2 cytoplasmic domain [1] does not seem to contribute to the neuronal plasticity of pain processing [10].

\section{Synapse Development and Regulation of Synaptic Plasticity}

In addition to the activation or inhibition of pathways that regulate cell shape or migration, Reelin signaling through Apoer 2 also induces the tyrosine phosphorylation of NMDA receptor (NR2) subunits, which changes the surface distribution and activity of this glutamate receptor and synaptic ion channel. This results in the Reelin-dependent regulation of synaptic plasticity over a wide physiological range, and this is further modulated by differential splicing of the Apoer 2 cytoplasmic domain [1]. The importance of Reelin signaling for NMDA receptor function is further emphasized by its role in the regulation of NMDA receptor maturation, which requires the participation of b1-integrins [11]. Moreover, Reelin has a powerful effect on the function of synapses in postmitotic neurons where it promotes dendritic spine development [12] and Apoer2 is required to ensure long-term neuronal survival in vivo [13]. LRP1 can also modulate NMDA receptor function through a tissue-type plasminogen activator dependent mechanism that involves the second NPxY motif in LRP1 [14]. 
The fundamental importance of ApoE receptors for synapse function and development is also illustrated by recent findings from two independent groups who revealed the mechanism by which LRP4 controls the development of a specialized peripheral synapse, the neuromuscular junction $[15,16]$. LRP4 deficient newborn mice completely lack neuromuscular junctions and consequently they are still-born. The underlying mechanism that is disrupted by the absence of LRP4 involves a role of LRP4 as an obligate coreceptor for the muscle specific tyrosine kinase MUSK and the neuronally produced ligand agrin. Binding of agrin to LRP4 induces clustering and transphosphorylation of MUSK. The latter does not bind agrin and therefore the neuron-to-muscle signal is disrupted in the absence of LRP4. As a result, acetylcholine receptors fail to form postsynaptic clusters, a process that requires MUSK activation (Figure $3)$.

Taken together, these independent findings have revealed distinct, but nevertheless molecularly similar mechanisms by which ApoE receptors function in neurons and which are critical for the formation or function of central as well as peripheral synapses.

\section{ApoE receptors and Alzheimer's Disease}

ApoE is the major risk factor/modifier of age of onset of late-onset Alzheimer's disease (AD). ApoE4 carriers develop AD in a dose-dependent manner on average up to 10 years earlier than the remainder of the population. Mechanisms that have been suggested by which ApoE exerts its strong isoform-specific effect on amyloid plaque formation include increased fibrillogenesis of b-amyloid, impaired clearance of the amyloid-b peptide from the extracellular space [17, 18] and diminished export across the blood-brain-barrier [19]. An alternative mechanism our laboratory has proposed involves a direct effect of ApoE on its own receptors, which interferes with the ability of these multifunctional ApoE receptors to signal to neurons, thereby impairing synaptic plasticity [1], spine formation [12], learning and memory [1] and neuronal survival [13]. Ab oligomers have been shown to induce synaptic dysfunction, which precedes neuronal loss in AD patients [20,21].

Another mechanism by which ApoE receptors can potently influence the production of bamyloid is by altering the subcellular trafficking of the amyloid precursor protein (APP) [22]. A large amount of evidence has accumulated over the last few years that shows that ApoE receptors can directly as well as indirectly alter the expression, distribution and routing of APP and thereby influence the pathways that control APP processing and thus Ab production. LRP1 regulates the expression of ApoE in the CNS while conversely disruption of APP and its close relative APLP2 increases LRP1 expression [23]. Upregulation of LRP1 expression is reversed by overexpression of the APP intracellular domain. Apoer 2 can increase Ab production by decreasing APP endocytosis through altering APP partitioning [24]. On the other hand, ApoE binding to Apoer2 increases APP endocytosis and Ab production in an isoform specific manner [25]. Intriguingly, nuclear liver $X$ receptor (LXR) agonists which regulate cholesterol homeostasis and which also have anti-inflammatory activity, reduce Ab production by increasing metalloproteinase dependent a-cleavage of APP as well as ectodomain release of Apoer2 through inhibition of metalloproteinase inhibitor TIMP-3 expression [26].

Another LDL receptor family member that has a major effect on the subcellular trafficking of APP and on its processing to b-amyloid is SORL1 (a.k.a. LR11) through interaction with intracellular adaptor proteins [27]. Loss of SORL1 in the mouse increases APP cleavage and $\mathrm{Ab}$ production $[28,29]$. Increased production of soluble APP ectodomain (APPs) increases ERK activation and neurogenesis [30]. Taken together, these findings emphasize the close functional relationship between ApoE receptors, APP and the regulation of the b-amyloid producing machinery, consistent with a model in which ApoE exerts its effect on AD age-ofonset through interaction with ApoE receptors. 


\section{Human Genetics}

The genes for all members of the LDL receptor gene family shown in Figure 1 have been disrupted in the mouse. Aside from the LDL receptor, where mutations cause familial hypercholesterolemia (FH), confirmed human gene defects have so far only been identified in LRP2 (a.k.a. Megalin) and VLDLR, while a sequence variant that is associated with coronary artery disease and myocardial infarction has been found in LRP8 [31]. Defects in LRP2 are the cause for Donnai-Barrow syndrome, a congenital malformation that includes agenesis of the corpus callosum, diaphragmatic hernia, facial dysmorphology, ocular anomalies, sensorineural hearing loss and developmental delay $[32,33]$. The defects seen in these patients are similar, but not identical to those seen in C57BL/6 $\times 129$ hybrid LRP2 deficient mice, indicating that genetic modifiers influence the relative developmental importance of this large ApoE receptor between different mammalian species and between different mouse strain backgrounds over a wide range.

VLDLR-deficiency in humans was first identified by Boycott et al. [34] in the Canadian Hutterite population by a combination of a gene mapping and candidate gene approach. Conversely to LRP2 deficiency, this fully autosomal recessive gene defect manifests itself also with a similar, but more severe phenotype in humans compared to the mouse. While the latter present with relatively mild cerebellar hypoplasia and primarily rostral cerebellar malformation with no recognizable ataxia, humans lacking VLDLR show severe cerebellar vermial hypolasia, accompanied by delayed motoric development and persistent truncal ataxia. Additional genetically distinct mutations in human VLDLR have been reported in independent pedigrees in Middle Eastern populations [35-37]. Neuroanatomically, the affected patients are indistinguishable from the initially reported Hutterites. By contrast, considerable variations between the two geographically separated populations, but also between individuals, are seen with respect to motor development. Several, but not all, affected individuals of a large Turkish pedigree were reported to be habitually quadrupedal, prompting the authors to attribute VLDLR as a crucial gene for the evolution of bipedal gait [36]. This conclusion has been drawn into question, however, since even within the same families this behavioral phenotype is not penetrant and the presence of adverse environmental and societal conditions offer far simpler and straightforward explanations $[38,39]$.

Recently, the first compound heterozygous case has been reported in a young child with developmental delay [40] where loss of VLDLR function is caused by two novel and genetically independent mutations in the VLDLR extracellular domain, resulting in premature translational truncation of the gene product from one allele and a missense mutation in the other, which matches a known FH causing mutation in LDLR, mostly likely through ER retention and degradation of the misfolded protein.

Other human autosomal recessive defects with phenotypes that are virtually identical, but nonallelic to, VLDLR deficiency have also been reported [36,37]. One of these loci has been narrowed to a short minimal region on Chr. 17 [37]. This region contains Crk, a downstream component of the Reelin signaling pathway [3,4] (Figure 2), prompting Boycott et al. [40] to propose Crk as a highly likely candidate gene for the $\mathrm{Chr} .17$ defect.

SORL1 (a.k.a. LR11) is an LDL receptor family member that combines most of the structural features of the core family members (Figure 1A) with functional domains found in other not related proteins, most notably a vacuolar protein sorting (VPS) domain [41]. Some of the in vivo functions of SORL1 resemble those of LRP1, in particular with respect to its role in regulating the trafficking of APP and as a signal modulator in the vascular wall, where it regulates AngII dependent smooth muscle cell migration [42]. SORL1 expression is dramatically reduced in the brains of AD patients [28] and intronic variants within SORL1 are 
genetically associated with AD [43]. Postmortem expression analysis also showed a statistically significant inverse correlation between SORL1 expression and cognitive impairment, suggesting SORL1 expression as a marker for prodromal AD [44]. Intriguingly, docosahexanoic acid, an essential omega-3 fatty acid, increases SORL1 expression in vitro and in mice in vivo [45], providing a potential mechanism for a role of diet in AD prevention.

LRP6 is a 'distant' member of the LDL receptor gene family and functions as an obligate component of the Wnt signaling complex at the plasma membrane which also includes Frizzled (Fz) receptors. A particular LRP6 variant (Ile1062Val) has previously been shown to have decreased canonical Wnt signaling associated with reduced bone mass. The same variant was found to convey an increased risk for late-onset $\mathrm{AD}$ in an ApoE4 dependent manner suggesting a role for Wnt signaling in $\mathrm{AD}$ pathogenesis [46].

\section{Conclusions}

Over the last decade the nervous system has morphed from a sideshow of lipoprotein receptor research to main stage act. ApoE receptors in particular have distinguished themselves as pivotal regulators of crucial developmental and synaptic processes that control neuronal migration and positioning, learning and memory, synaptogenesis and neuronal survival. As we continue to peel away the layers that shroud their increasingly complex functions in the central nervous system, we may ultimately arrive at a comprehensive understanding of the fundamental roles of this remarkable, multifunctional and ancient gene family in the maintenance and operation of our most complex organ, the brain.

\section{Acknowledgments}

I am indebted to all present and former members of my laboratory and of the Department of Molecular Genetics and to Nancy Heard for artwork. I also explicitly acknowledge the many colleagues in the field whose work could not be included or discussed in the depth it clearly deserves. I acknowledge present and previous support from the National Institutes of Health, the Alexander-von-Humboldt Foundation, the American Heart Association, the Alzheimer's Association and the University of Texas Southwestern Medical Center.

\section{References}

1. Herz J, Chen Y. Reelin, lipoprotein receptors and synaptic plasticity. Nat Rev Neurosci 2006;7:850859. [PubMed: 17053810]

2. Zhang G, Assadi AH, McNeil RS, et al. The Pafah1b complex interacts with the Reelin receptor VLDLR. PLoS ONE 2007;2:e252. [PubMed: 17330141] This paper reveals a novel interaction between the Pafah1b complex and a highly conserved region within the cytoplasmic domain of VLDLR. A single amino acid difference between VLDLR and Apoer2 determines specificity and suggests that VLDLR non-redundantly activates an intracellular signaling branch that is separate from Apoer2.

3. Matsuki T, Pramatarova A, Howell BW. Reduction of Crk and CrkL expression blocks reelin-induced dendritogenesis. J Cell Sci 2008;121:1869-1875. [PubMed: 18477607]

4. Park TJ, Curran T. Crk and Crk-like play essential overlapping roles downstream of disabled-1 in the Reelin pathway. J Neurosci 2008;28:13551-13562. [PubMed: 19074029] Refs 3 and 4 use genetic approaches to demonstrate important roles for Crk and CrkL during neuronal migration and dendrite formation.

5. Chai X, Forster E, Zhao S, et al. Reelin stabilizes the actin cytoskeleton of neuronal processes by inducing n-cofilin phosphorylation at serine3. J Neurosci 2009;29:288-299. [PubMed: 19129405] This paper establishes N-cofilin as a novel intracellular target of Reelin signaling, through SFK-mediated activation of LIM kinase.

6. Blake SM, Strasser V, Andrade N, et al. Thrombospondin-1 binds to ApoER2 and VLDL receptor and functions in postnatal neuronal migration. Embo J 2008;27:3069-3080. [PubMed: 18946489] This 
study reveals Thrombospondin as a novel ligand for Apoer2 and Vldlr that regulates the formation of the rostral migratory stream.

7. Larouche M, Beffert U, Herz J, Hawkes R. The Reelin receptors Apoer2 and Vldlr coordinate the patterning of Purkinje cell topography in the developing mouse cerebellum. PLoS ONE 2008;3:e1653. [PubMed: 18301736] The study reveals non-redundant functions for Apoer2 and Vldlr that coordinately control the architecture of the cerebellum.

8. Hack I, Hellwig S, Junghans D, et al. Divergent roles of ApoER2 and Vldlr in the migration of cortical neurons. Development 2007;134:3883-3891. [PubMed: 17913789] Non-redundant roles for Vldlr and Apoer2 in the positioning and migration of cortical neurons are revealed.

9. Hashimoto-Torii K, Torii M, Sarkisian MR, et al. Interaction between Reelin and Notch signaling regulates neuronal migration in the cerebral cortex. Neuron 2008;60:273-284. [PubMed: 18957219] Reelin signaling modulates Notch processing and conversely, overexpression of Notch ICD mitigates the neuronal migration defect in reeler mice.

10. Akopians AL, Babayan AH, Beffert U, et al. Contribution of the Reelin signaling pathways to nociceptive processing. Eur J Neurosci 2008;27:523-537. [PubMed: 18279306] This study distinguishes between Reelin and ApoE receptor dependent signaling pathways that differentially control synaptic plasticity and nociception, respectively.

11. Groc L, Choquet D, Stephenson FA, et al. NMDA receptor surface trafficking and synaptic subunit composition are developmentally regulated by the extracellular matrix protein Reelin. J Neurosci 2007;27:10165-10175. [PubMed: 17881522] Synaptic and extrasynaptic localization and functional maturation of NMDA receptors is shown to be regulated by Reelin. Reelin accumulation at the synyapse coincides with postnatal NMDA receptor maturation.

12. Niu S, Yabut O, D'Arcangelo G. The Reelin signaling pathway promotes dendritic spine development in hippocampal neurons. J Neurosci 2008;28:10339-10348. [PubMed: 18842893]

13. Beffert U, Nematollah Farsian F, Masiulis I, et al. ApoE receptor 2 controls neuronal survival in the adult brain. Curr Biol 2006;16:2446-2452. [PubMed: 17174920]

14. Martin AM, Kuhlmann C, Trossbach S, et al. The functional role of the second NPXY motif of the LRP1 beta-chain in tissue-type plasminogen activator-mediated activation of N-methyl-D-aspartate receptors. J Biol Chem 2008;283:12004-12013. [PubMed: 18321860]

15. Kim N, Stiegler AL, Cameron TO, et al. Lrp4 is a receptor for Agrin and forms a complex with MuSK. Cell 2008;135:334-342. [PubMed: 18848351]

16. Zhang B, Luo S, Wang Q, et al. LRP4 serves as a coreceptor of agrin. Neuron 2008;60:285-297. [PubMed: 18957220] References 15 and 16 solve a long-time mystery of neuromuscular junction development, by revealing the identity of the long-sought MUSK co-receptor as LRP4.

17. Jiang Q, Lee CY, Mandrekar S, et al. ApoE promotes the proteolytic degradation of Abeta. Neuron 2008;58:681-693. [PubMed: 18549781] ApoE is shown to regulate amyloid $\beta$ degradation dependent upon lipidation status. LXR agonists are shown to increase ApoE lipidation and accelerate ApoEdependent Ab degradation.

18. Nazer B, Hong S, Selkoe DJ. LRP promotes endocytosis and degradation, but not transcytosis, of the amyloid-beta peptide in a blood-brain barrier in vitro model. Neurobiol Dis 2008;30:94-102. [PubMed: 18289866]

19. Deane R, Sagare A, Hamm K, et al. apoE isoform-specific disruption of amyloid beta peptide clearance from mouse brain. J Clin Invest 2008;118:4002-4013. [PubMed: 19033669] References 18 and 19 reveal roles for lipoprotein receptors in the transcytosis and degradation of $\mathrm{Ab}$ at the bloodbrain-barrier.

20. Shankar GM, Bloodgood BL, Townsend M, et al. Natural oligomers of the Alzheimer amyloid-beta protein induce reversible synapse loss by modulating an NMDA-type glutamate receptor-dependent signaling pathway. J Neurosci 2007;27:2866-2875. [PubMed: 17360908]

21. Shankar GM, Li S, Mehta TH, et al. Amyloid-beta protein dimers isolated directly from Alzheimer's brains impair synaptic plasticity and memory. Nat Med 2008;14:837-842. [PubMed: 18568035] Refs 20 and 21 show how naturally produced Ab oligomers, and oligomers isolated from the brains of Alzheimer's disease patients affects synaptic plasticity in the hippocampus.

22. Marzolo MP, Bu G. Lipoprotein receptors and cholesterol in APP trafficking and proteolytic processing, implications for Alzheimer's disease. Semin Cell Dev Biol. 2008 A review article that 
puts the role of lipoprotein receptors in the trafficking and processing of APP into a pathophysiological perspective.

23. Liu Q, Zerbinatti CV, Zhang J, et al. Amyloid precursor protein regulates brain apolipoprotein $\mathrm{E}$ and cholesterol metabolism through lipoprotein receptor LRP1. Neuron 2007;56:66-78. [PubMed: 17920016] The ICD of APP is shown to regulate LRP1 expression, which in turn affects ApoE and cholesterol levels in the CNS.

24. Fuentealba RA, Barria MI, Lee J, et al. ApoER2 expression increases Abeta production while decreasing Amyloid Precursor Protein (APP) endocytosis: Possible role in the partitioning of APP into lipid rafts and in the regulation of gamma-secretase activity. Mol Neurodegener 2007;2:14. [PubMed: 17620134]

25. He X, Cooley K, Chung CH, et al. Apolipoprotein receptor 2 and X11 alpha/beta mediate apolipoprotein E-induced endocytosis of amyloid-beta precursor protein and beta-secretase, leading to amyloid-beta production. J Neurosci 2007;27:4052-4060. [PubMed: 17428983]

26. Hoe HS, Cooper MJ, Burns MP, et al. The metalloprotease inhibitor TIMP-3 regulates amyloid precursor protein and apolipoprotein E receptor proteolysis. J Neurosci 2007;27:10895-10905. [PubMed: 17913923]

27. Schmidt V, Sporbert A, Rohe M, et al. SorLA/LR11 regulates processing of amyloid precursor protein via interaction with adaptors GGA and PACS-1. J Biol Chem 2007;282:32956-32964. [PubMed: 17855360]

28. Andersen OM, Reiche J, Schmidt V, et al. Neuronal sorting protein-related receptor sorLA/LR11 regulates processing of the amyloid precursor protein. Proc Natl Acad Sci U S A 2005;102:1346113466. [PubMed: 16174740]

29. Dodson SE, Andersen OM, Karmali V, et al. Loss of LR11/SORLA enhances early pathology in a mouse model of amyloidosis: evidence for a proximal role in Alzheimer's disease. J Neurosci 2008;28:12877-12886. [PubMed: 19036982]

30. Rohe M, Carlo AS, Breyhan H, et al. Sortilin-related receptor with A-type repeats (SORLA) affects the amyloid precursor protein-dependent stimulation of ERK signaling and adult neurogenesis. J Biol Chem 2008;283:14826-14834. [PubMed: 18362153] References 27-30 demonstrate the powerful role of SORL1 in the trafficking and processing of APP and investigate its pathophysiological importance in in vivo models.

31. Shen GQ, Li L, Girelli D, et al. An LRP8 variant is associated with familial and premature coronary artery disease and myocardial infarction. Am J Hum Genet 2007;81:780-791. [PubMed: 17847002] A frequent coding SNP in the cytoplasmic domain of ApoER2/LRP8 is identified as a novel risk factor for coronary artery disease in humans.

32. Kantarci S, Al-Gazali L, Hill RS, et al. Mutations in LRP2, which encodes the multiligand receptor megalin, cause Donnai-Barrow and facio-oculo-acoustico-renal syndromes. Nat Genet 2007;39:957959. [PubMed: 17632512]

33. Kantarci S, Ragge NK, Thomas NS, et al. Donnai-Barrow syndrome (DBS/FOAR) in a child with a homozygous LRP2 mutation due to complete chromosome 2 paternal isodisomy. Am J Med Genet A 2008;146A:1842-1847. [PubMed: 18553518] Refs 32 and 33 identify novel mutations in LRP2 as the cause for Donnai-Barrow Syndrome, a congenital abnormality in humans.

34. Boycott KM, Flavelle S, Bureau A, et al. Homozygous deletion of the very low density lipoprotein receptor gene causes autosomal recessive cerebellar hypoplasia with cerebral gyral simplification. Am J Hum Genet 2005;77:477-483. [PubMed: 16080122]

35. Moheb LA, Tzschach A, Garshasbi M, et al. Identification of a nonsense mutation in the very lowdensity lipoprotein receptor gene (VLDLR) in an Iranian family with dysequilibrium syndrome. Eur J Hum Genet 2008;16:270-273. [PubMed: 18043714]

36. Ozcelik T, Akarsu N, Uz E, et al. Mutations in the very low-density lipoprotein receptor VLDLR cause cerebellar hypoplasia and quadrupedal locomotion in humans. Proc Natl Acad Sci U S A 2008;105:4232-4236. [PubMed: 18326629]

37. Turkmen S, Hoffmann K, Demirhan O, et al. Cerebellar hypoplasia, with quadrupedal locomotion, caused by mutations in the very low-density lipoprotein receptor gene. Eur J Hum Genet 2008;16:1070-1074. [PubMed: 18364738] Refs 35-37 identify novel mutations in VLDLR with a high incidence of quadrupedal locomotion in Middle Eastern populations. 
38. Herz J, Boycott KM, Parboosingh JS. "Devolution" of bipedality. Proc Natl Acad Sci U S A 2008;105:E25. [PubMed: 18487453]

39. Humphrey N, Mundlos S, Turkmen S. Genes and quadrupedal locomotion in humans. Proc Natl Acad Sci U S A 2008;105:E26. [PubMed: 18483196] Refs 38 and 39 offer alternative explanations for the high incidence of quadrupedal gait in Middle Eastern patients afflicted with disequilibrium syndrome.

40. Boycott KM, Bonnemann C, Herz J, et al. Mutations in VLDLR as a Cause for Autosomal Recessive Cerebellar Ataxia with Mental Retardation (Dysequilibrium Syndrome). J. Child Neurol. 2009 in press. This paper describes the first compound heterozygous case of disequilibrium syndrome caused by independent loss of function mutations in VLDLR.

41. Willnow TE, Petersen CM, Nykjaer A. VPS10P-domain receptors - regulators of neuronal viability and function. Nat Rev Neurosci 2008;9:899-909. [PubMed: 19002190] An excellent review on the functions of VPS10 domain containing receptors, including SORL1/LR11, and their role in the nervous system.

42. Jiang M, Bujo H, Ohwaki K, et al. Ang II-stimulated migration of vascular smooth muscle cells is dependent on LR11 in mice. J Clin Invest 2008;118:2733-2746. [PubMed: 18618022] This paper reveals a novel function for SORLA/LR11 as a signal modulator in the smooth muscle cells of the vascular wall.

43. Rogaeva E, Meng Y, Lee JH, et al. The neuronal sortilin-related receptor SORL1 is genetically associated with Alzheimer disease. Nat Genet 2007;39:168-177. [PubMed: 17220890]

44. Sager KL, Wuu J, Leurgans SE, et al. Neuronal LR11/sorLA expression is reduced in mild cognitive impairment. Ann Neurol 2007;62:640-647. [PubMed: 17721864] Refs 43 and 44 provide evidence for a neuroprotective role of SORL1/LR11 in the human brain.

45. Ma QL, Teter B, Ubeda OJ, et al. Omega-3 fatty acid docosahexaenoic acid increases SorLA/LR11, a sorting protein with reduced expression in sporadic Alzheimer's disease (AD): relevance to AD prevention. J Neurosci 2007;27:14299-14307. [PubMed: 18160637]

46. De Ferrari GV, Papassotiropoulos A, Biechele T, et al. Common genetic variation within the lowdensity lipoprotein receptor-related protein 6 and late-onset Alzheimer's disease. Proc Natl Acad Sci U S A 2007;104:9434-9439. [PubMed: 17517621] A functional variant of LRP6 genetically interacts with ApoE4 in late-onset Alzheimer's disease, implicating Wnt signaling in the disease pathogenesis.

47. Herz J, Chen Y, Masiulis I, Zhou L. Expanding Functions of Lipoprotein Receptors. J. Lipid. Res. 2009 in press. 


\begin{tabular}{|c|c|}
\hline$\sum_{0}^{0}$ & $\begin{array}{l}\text { Ligand binding type repeat } \\
\text { EGF precursor } \\
\text { homology domain }\end{array}$ \\
\hline & $\begin{array}{l}\text { VPS } 10 \text { homology } \\
\text { domain }\end{array}$ \\
\hline 0 & Fibronectin Repeat \\
\hline ○ & EGF Repeat \\
\hline 0 & O-linked sugar domain \\
\hline 】 & Transmembrane domain \\
\hline * & NPxY Motif \\
\hline
\end{tabular}

LDLR VLDLR Apo ER2

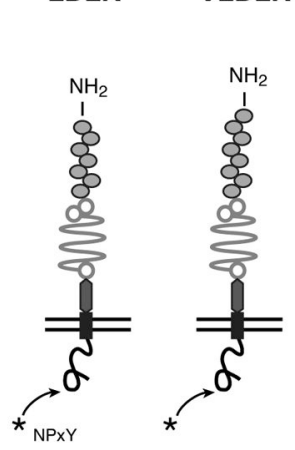

A

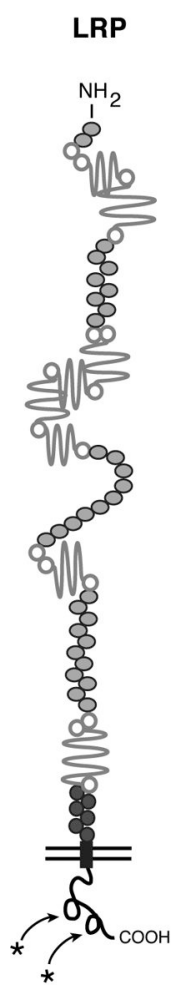

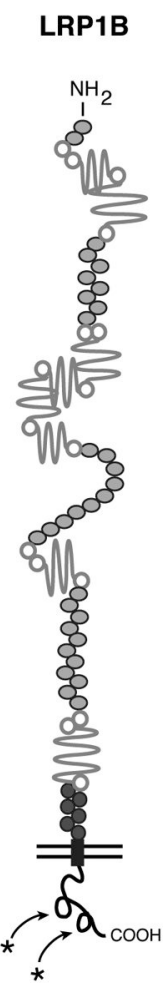

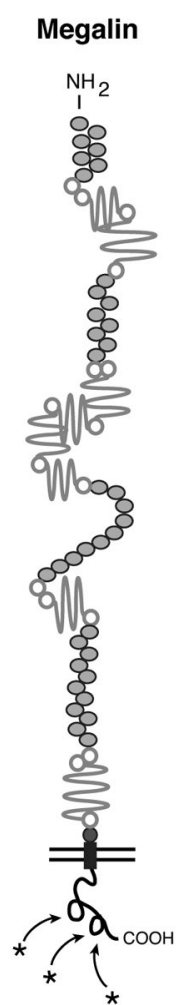

B

LRP5

LRP6

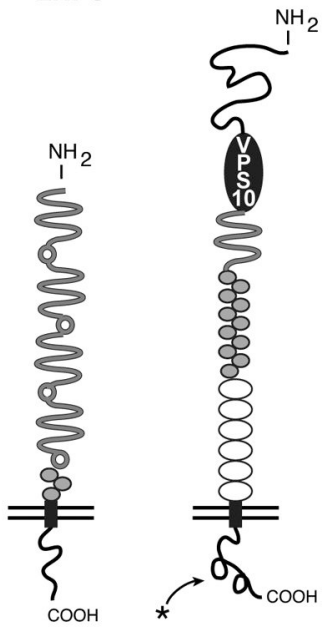

Figure 1. The LDL receptor family of ApoE receptors (modified from 1, 47)

Panel $A$, the core of the family in mammals. Panel B, physiologically and functionally important, related family members that do not share all the structural requirements of the "core members', including a ligand binding domain of variable repeat length at the amino terminus, followed by an EGF precursor homology ( $\beta$-propeller) domain, which in turn is followed by either another ligand binding domain, an O-linked sugar domain, or a ligand binding segment. All receptors are type I transmembrane proteins that are anchored by a single transmembrane segment. Core members contain short cytoplasmic tails with at least one 'NPxY' tetraamino acid motif that combines endocytosis with adapter protein docking functions. 


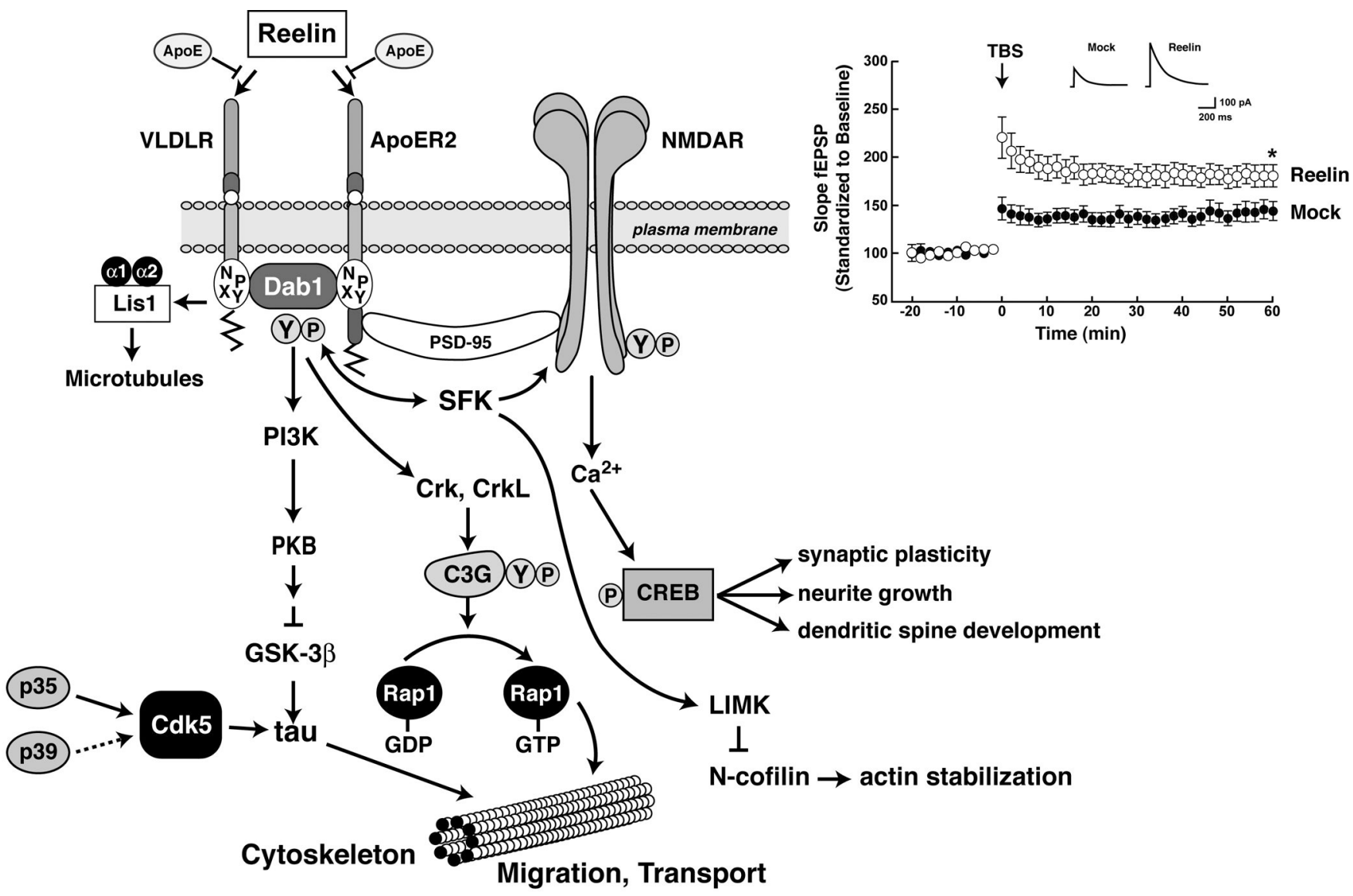

Figure 2. Vldlr and Apoer2 function as signal transducers in neurons (modified from 1, 47) Panel $A$, The large oligomeric high affinity signaling protein Reelin binds to Vldlr and Apoer2 and induces receptor clustering. Transactivation of Src family tyrosine kinases (SFK) results in phosphorylation and thereby activation of Disabled-1 (Dab1), an adaptor protein that interacts with NPxY motifs in both receptor tails. This activates phosphatidylinositol 3-kinase $(\mathrm{PI} 3 \mathrm{~K})$ and protein kinase $\mathrm{B}(\mathrm{PKB}, \mathrm{Akt})$, which in turn inhibits glycogen synthase kinase-3 $\beta$ (GSK3 $\beta$ ) and reduces phosphorylation of the microtubule stabilizing protein tau. Crk and Crklike (CrkL) function downstream of Dab1, regulating actin cytoskeleton rearrangement. Actin depolymerization is also controlled by LIM kinase (LIMK) which inhibits n-cofilin, an actin depolymerizing protein. Lis1 binds to tyrosine-phosphorylated Dab and as part of a Pafah1b complex regulates microtubule functions. Cyclin-dependent kinase 5 (Cdk5) and its activators p35 and p39 function parallel to Reelin. In the synapse, Apoer2 interacts with PSD95 through its cytoplasmic exon 19, thereby functionally coupling the Reelin signaling complex to the NMDA receptor (NMDAR). Reelin-activated SFKs phosphorylate the NMDA receptor NR2 subunits, thereby potentiating NMDAR-mediated $\mathrm{Ca}^{2+}$ influx. Phosphorylation of survival factor cAMP-response element binding protein (CREB) ensues. This phosphorylation event initiates the expression of genes that are important for synaptic plasticity, neurite growth and dendritic spine development.

Panel B, Reelin potentiates long-term potentiation (LTP) and enhances NMDAR-mediated currents in wild type CA1 pyramidal neurons. 

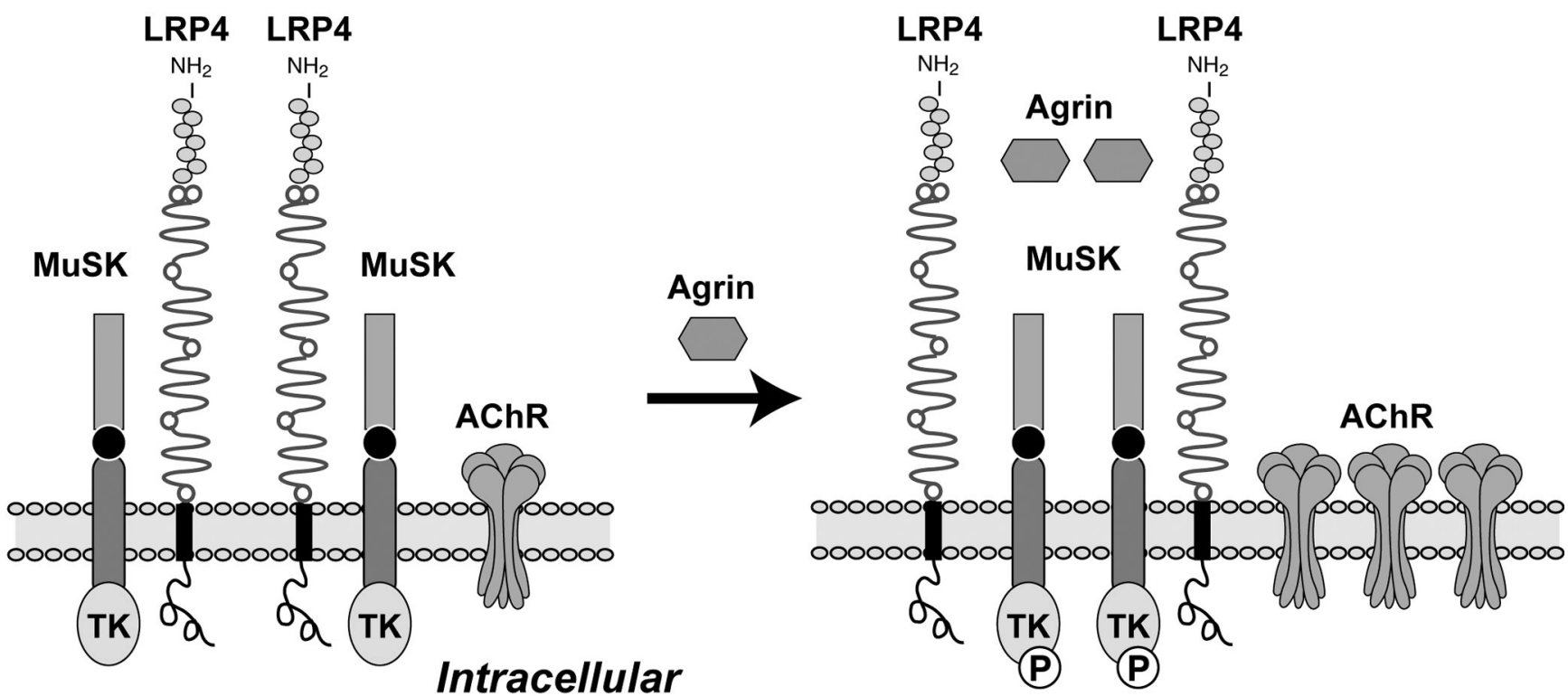

Figure 3. LRP4 is a receptor for agrin and a coreceptor for the tyrosine kinase MUSK in muscle (modified from 47)

The membrane tyrosine kinase MUSK is required for acetylcholine receptor (AchR) clustering in neuromuscular endplates. However, MUSK does not bind the obligate signaling protein agrin directly. Instead, MUSK must form a complex with LRP4, which is a receptor for agrin. This in turn enhances complex formation of MUSK with LRP4 and induces transphosphorylation and activation of MUSK, followed by AChR clustering. 